\title{
KLOTHO Gene Polymorphism Is Associated With Coronary Artery Stenosis but Not With Coronary Calcification in a Korean Population
}

\author{
Sang-Ho Jo, ${ }^{1 \dagger} \mathrm{MD}$, Seong-Gyun KIM, ${ }^{2,3 \dagger} \mathrm{MD}$, \\ Young Jin Chol, ${ }^{1} \mathrm{MD}$, Na-Rae Joo, ${ }^{1} \mathrm{MD}$, Goo-Yeong Cho, ${ }^{1} \mathrm{MD}$, \\ Sun-Ryoung CHOI, ${ }^{1} \mathrm{MD}$, Eun-Jung KIM, ${ }^{2,3}$ MD, Hyun-Sook KIM, ${ }^{1} \mathrm{MD}$, \\ Hyung-Jik KIM, ${ }^{2,3} \mathrm{MD}$, and Chong-Yun RHIM, ${ }^{1}$ MD
}

SUMMARY

Experimental studies have demonstrated KLOTHO gene polymorphism might be associated with vascular atherosclerosis and calcification. However, the impact of this genetic variant on human coronary arteries still remains to be elucidated. We investigated the effect of a KLOTHO gene variant on coronary artery stenosis and calcification. Four hundred and thirty-four patients referred for chest pain were enrolled. All the patients underwent coronary angiography and were investigated for polymorphism of the KLOTHO G395A gene. Coronary artery disease (CAD) was defined as $\geq 50 \%$ diameter stenosis in at least one coronary artery. The other patients were considered to be controls. Homozygotes or heterozygotes for G395A were significantly more common in the CAD patients than in the controls $(30.2 \%$ versus $21.5 \%, P=0.039)$. In the subgroup aged $<60$ years, the G395A mutant was more frequent in CAD than in control $(35.3 \%$ versus $18.8 \%, P$ $=0.016)$, but in patients $\geq 60$ years, there was no difference $(28.0 \%$ versus $24.1 \%, P=$ 0.473). Using multivariate analysis, we identified the KLOTHO gene G395A mutant as an independent risk factor of CAD (OR 1.712, 95\% CI [1.066-2.749], $P=0.026$ ). The frequency of the KLOTHO gene G395A mutant was not different between the calcified and noncalcified coronary artery groups $(25.7 \%, 26.4 \%$, respectively, $P=0.861)$ and an A allele carrier state was not an independent risk factor of coronary artery calcification. In conclusion, the KLOTHO gene G395A allele carrier state may be associated with CAD but not with coronary artery calcification in this Korean population. (Int Heart J 2009; 50: 23-32)

Key words: Klotho, Gene, Coronary artery, Atherosclerosis, Stenosis, Calcification

From the Divisions of ${ }^{1}$ Cardiology and ${ }^{2}$ Nephrology, Department of Internal Medicine, Hallym University Sacred Heart Hospital, Anyang-si, Gyeonggi-do, and ${ }^{3}$ Kidney Research Institute, Hallym University College of Medicine, Chunchon-si, Gangwon-do, South Korea.

Authors that contributed equally to this study.

Address for correspondence: Young-Jin Choi, MD, Division of Cardiology, Department of Internal Medicine, Hallym University Sacred Heart Hospital, 896, Pyeongchon-dong, Dongan-gu, Anyang-si, Gyeonggi-do, 431-070, South Korea.

Received for publication July 28, 2008.

Revised and accepted September 25, 2008. 
I $\mathrm{T}$ has been demonstrated that the overall biological effect of KLOTHO is suppression of the aging process and an over-expressed KLOTHO gene has been shown to increase the life span of mice. ${ }^{1)}$ The KLOTHO gene defect in an experimental mouse model was reported to have similar expressions of premature aging including osteoporosis, infertility, gonadal, thymus, and skin atrophy, physical inactivity, and pulmonary emphysema. ${ }^{2)}$ Of note, they displayed progressive vascular atherosclerosis and calcification. ${ }^{2)}$ Subsequent human studies indicated that the functional variant of KLOTHO (KL-VS) may be associated with longevity and early onset occult coronary artery disease (CAD). ${ }^{3-5)}$ Furthermore, another report described the association of this variant of the KLOTHO gene with high-density lipoprotein cholesterol (HDL), systolic blood pressure, and stroke. ${ }^{4}$ Recently, G395A in the promoter region was suggested to be significantly linked with bone mineral density, which appeared to be related with arterial atherosclerosis and calcification. ${ }^{6,7)}$

However, no study has investigated the association between KLOTHO gene mutation and coronary calcification. Most research has dealt with only coronary atherosclerotic disease and osteoporosis, both of which may potentially influence coronary calcification.

One study conducted in Japan involving 197 patients demonstrated that the incidence of A allele carriers of the KLOTHO gene was significantly higher in the CAD group than in the control group (29.9\% versus $19.0 \%)$ and the A allele was an independent predictor of CAD. ${ }^{8)}$ However, another study concluded KLOLTHO G395A polymorphism had no relation with CAD in a Korean population. ${ }^{9)}$ Therefore, it has become imperative to evaluate the real contribution of the KLOTHO gene variant to CAD risk and the association between KLOTHO and coronary arterial calcification.

\section{Methods}

Study population: Between November 2004 and August 2005, a total of 434 consecutive Korean patients aged 19 to 97 years (mean age, 61.8 years) who underwent coronary angiography (CAG) due to chest pain were enrolled in this study. The exclusion criteria were as follows; pregnancy, lactation, history of hypersensitivity reaction to contrast media, cardiogenic shock, pulmonary edema, mechanical ventilation, and use of metformin within 48 hours of the procedure.

This study was approved by the institutional review board at our hospital and all participating patients provided informed consent.

Coronary angiography: Coronary angiography was performed routinely, via a femoral or radial approach in all patients. Coronary artery disease was defined as $\geq 50 \%$ diameter stenosis compared to the reference vessel diameter with vis- 
ual estimation by the 2 experts in interventional cardiology at our hospital, Drs. YJ Choi and SH Jo. Patients who had a diameter stenosis of $<50 \%$ at all coronary arteries served as controls. Coronary calcification was defined as positive if a calcified coronary artery was observed in at least one coronary artery segment in multigonal fluoroscopic and cine-angiographic images by 2 experts who were blind to the genotype of the patient. Coronary arterial intervention for a significant stenotic lesion was performed at the discretion of the operator.

Genotyping of KLOTHO G395A: Blood samples were obtained immediately after the vascular access prior to the coronary catheterization. Genomic DNA was extracted from samples of these peripheral blood mononuclear cells. The KLOTHO genotype (G395A) was determined using the TaqMan PCR assay. The sense oligonucleotide primer was TAGGGCCCGGCAGGAT and the antisense primer was CCTGGAGCGGCTTCGTC. The products were analyzed using an automated sequencer (ABI PRISM 7900HT, Applied Biosystems, Foster City, CA). Genotyping was performed and determined in a blinded fashion by laboratory personnel at Hallym University Sacred Heart Hospital.

Statistical analysis: Hardy-Weinberg equilibrium was tested with the chi-square test. Data were compared using the chi-square test or Fisher's exact test (categorical variables) and the Student $t$ test (continuous variables). $P$ values $<0.05$ were considered statistically significant. To assess the effects of various environmental and genetic factors on the presence of coronary artery stenosis and calcification, the odds ratios (ORs) and $95 \% \mathrm{CI}$ for all potential risk factors related to it were calculated by multiple logistic regression analysis. For the multivariate model, hypertension, diabetes, and dyslipidemia along with sex, smoking, and G395A genotype were incorporated into the model as categorical variables, whereas age and basal serum creatinine were expressed as a continuous variable. All statistical analyses were performed using SPSS software version 13.0 (SPSS, Inc.).

\section{Results}

Patient population and baseline characteristics: A total of 434 patients referred for diagnostic or interventional coronary angiography due to chest pain were enrolled. All of the patients underwent coronary angiography and were analyzed for the KLOTHO G395A genotype. Demographic and baseline data according to the coronary artery disease status are provided in Table I. Older patients, males, hypertension, diabetes, and smoking were more common in CAD patients than in the control group, and the left ventricular ejection fraction (LVEF) and total cholesterol level were also significantly different between the two groups (Table I). 
Genotype distribution: The distribution of genotypes of the polymorphisms according to the study group is presented in Table II. The allele frequency of KLOTHO G395A polymorphism was $85.4 \%$ for the $\mathrm{G}$ allele and $14.6 \%$ for the A allele. The genotypic distributions of this polymorphic region in both groups were within Hardy-Weinberg equilibrium.

Association of KLOTHO G395A polymorphism with coronary artery disease: The genotype distribution of the G395A polymorphism according to study group is shown in Table II. GA and AA mutants were higher in the CAD group, although the difference was not statistically significant $(P=0.116)$. However, the incidence of total -395A allele carriers (heterozygotes + homozygotes) was significantly greater in the CAD patients than in the control $(30.2 \%$ versus $21.5 \%, P=$ 0.039) (Table III).

Notably, in the subgroup $<60$ years old, the $-395 \mathrm{~A}$ allele carrier was more common in the CAD group (35.3\% versus $18.8 \%, P=0.016)$ (Table IV), while there was no difference between the 2 groups $(28.0 \%$ versus $24.1 \%$, respectively, $P=0.473)$ in the subgroup $\geq 60$ years old. When employing more stringent cri-

Table I. Baseline Characteristics of the Patients

\begin{tabular}{lccr}
\hline Characteristic & CAD $(n=225)$ & Control $(n=209)$ & $P$ \\
\hline Age, years ${ }^{*}$ & $64.1 \pm 11.5$ & $59.3 \pm 12.1$ & $<0.001$ \\
Sex, male/female & $139 / 86$ & $103 / 106$ & 0.009 \\
Weight, kg & $63.9 \pm 10.4$ & $64.5 \pm 11.0$ & 0.564 \\
BMI, kg/m 2 $^{*}$ & $24.5 \pm 3.0$ & $24.7 \pm 3.2$ & 0.412 \\
LVEF, \% & $54.9 \pm 10.9$ & $58.3 \pm 11.2$ & 0.003 \\
Diabetes mellitus (\%) & $82(36.4)$ & $56(26.8)$ & 0.031 \\
Hypertension (\%) & $141(62.7)$ & $111(53.1)$ & 0.044 \\
Dyslipidemia (\%) & $97(44.1)$ & $76(37.3)$ & 0.152 \\
Current smoker (\%) & $64(28.4)$ & $38(18.2)$ & 0.012 \\
Baseline SCr, mg/dL & $1.16 \pm 1.04$ & $1.00 \pm 0.97$ & 0.119 \\
Total cholesterol, mg/dL & $175.6 \pm 39.8$ & $183.8 \pm 38.8$ & 0.034 \\
LDL cholesterol, mg/dL ${ }^{*}$ & $118.2 \pm 39.8$ & $119.7 \pm 35.7$ & 0.694 \\
C-reactive protein, mg/L & $9.07 \pm 21.0$ & $10.7 \pm 26.9$ & 0.535 \\
\hline
\end{tabular}

CAD indicates coronary artery disease, defined as $\geq 50 \%$ diameter stenosis compared with reference vessel diameter by coronary angiography; $\mathrm{BMI}$, body mass index; $\mathrm{CrCl}$, creatinine clearance; and LVEF, left ventricular ejection fraction.

*Values are mean \pm standard deviation.

Table II. Allele Frequencies of KLOTHO G395A in Coronary Artery Disease and Control Groups

\begin{tabular}{lccc}
\hline & CAD $(n=225)$ & Control $(n=209)$ & $P$ \\
\hline GG & $157(69.7 \%)$ & $164(78.5 \%)$ & \\
GA & $60(26.7 \%)$ & $39(18.7 \%)$ & 0.116 \\
AA & $8(3.6 \%)$ & $6(2.9 \%)$ & \\
\hline
\end{tabular}

CAD indicates coronary artery disease, defined as $\geq 50 \%$ diameter stenosis compared with reference vessel diameter by coronary angiography. 
teria for coronary artery disease with the definition of diameter stenosis $\geq 75 \%$, there were significantly more $-395 \mathrm{~A}$ allele carriers in the CAD patients than in the controls for the subgroup $<60$ years of age $(34.8 \%$ versus $19.4 \%, P=0.025)$,

Table III. Association of KLOTHO G395A With Coronary Artery Disease

\begin{tabular}{lccc}
\hline & CAD $(n=225)$ & Control $(n=209)$ & $P$ \\
\hline GG & $157(69.8 \%)$ & $164(78.5 \%)$ & 0.039 \\
GA+AA & $68(30.2 \%)$ & $45(21.5 \%)$ & \\
\hline
\end{tabular}

CAD indicates coronary artery disease, defined as $\geq 50 \%$ diameter stenosis compared with reference vessel diameter by coronary angiography.

Table IV. Subgroup Analysis of Association of KLOTHO G395A With Coronary Artery Disease

\begin{tabular}{|c|c|c|c|}
\hline & CAD & Control & $P$ \\
\hline Age $<60$ years $(n=169)$ & & & 0.016 \\
\hline GG & $44(64.7 \%)$ & $82(81.2 \%)$ & \\
\hline $\mathrm{GA}+\mathrm{AA}$ & $24(35.3 \%)$ & $19(18.8 \%)$ & \\
\hline Age $\geq 60$ years $(n=265)$ & & & 0.473 \\
\hline GG & $113(72.0 \%)$ & $56(68.3 \%)$ & \\
\hline $\mathrm{GA}+\mathrm{AA}$ & $44(28.0 \%)$ & $26(31.7 \%)$ & \\
\hline Male $(n=242)$ & & & 0.075 \\
\hline GG & $95(68.3 \%)$ & $81(78.6 \%)$ & \\
\hline $\mathrm{GA}+\mathrm{AA}$ & $44(31.7 \%)$ & $22(21.4 \%)$ & \\
\hline Female $(n=192)$ & & & 0.320 \\
\hline GG & $62(72.1 \%)$ & $83(78.3 \%)$ & \\
\hline $\mathrm{GA}+\mathrm{AA}$ & $24(27.9 \%)$ & $23(21.7 \%)$ & \\
\hline Diabetes $(n=138)$ & & & 0.622 \\
\hline GG & $63(76.8 \%)$ & $45(80.4 \%)$ & \\
\hline $\mathrm{GA}+\mathrm{AA}$ & $19(23.2 \%)$ & $11(19.6 \%)$ & \\
\hline Nondiabetes $(n=296)$ & & & 0.021 \\
\hline GG & $94(65.7 \%)$ & $119(77.8 \%)$ & \\
\hline $\mathrm{GA}+\mathrm{AA}$ & $49(34.3 \%)$ & $34(22.2 \%)$ & \\
\hline Hypertension $(n=252)$ & & & 0.550 \\
\hline GG & $102(72.3 \%)$ & $84(75.7 \%)$ & \\
\hline $\mathrm{GA}+\mathrm{AA}$ & $39(27.7 \%)$ & $27(24.3 \%)$ & \\
\hline Nonhypertension $(n=182)$ & & & 0.013 \\
\hline GG & $55(65.5 \%)$ & $80(81.6 \%)$ & \\
\hline $\mathrm{GA}+\mathrm{AA}$ & $29(34.5 \%)$ & $18(18.4 \%)$ & \\
\hline Dyslipidemia $(n=173)$ & & & 0.306 \\
\hline GG & $70(72.2 \%)$ & $60(78.9 \%)$ & \\
\hline $\mathrm{GA}+\mathrm{AA}$ & $27(27.8 \%)$ & $16(21.1 \%)$ & \\
\hline Nondyslipidemia $(n=251)$ & & & 0.078 \\
\hline GG & $84(68.3 \%)$ & $100(78.1 \%)$ & \\
\hline $\mathrm{GA}+\mathrm{AA}$ & $39(31.7 \%)$ & $28(21.9 \%)$ & \\
\hline Smoking $(n=102)$ & & & 0.112 \\
\hline GG & $47(73.4 \%)$ & $33(86.8 \%)$ & \\
\hline $\mathrm{GA}+\mathrm{AA}$ & $17(26.6 \%)$ & $5(13.2 \%)$ & \\
\hline Nonsmoking $(n=332)$ & & & 0.091 \\
\hline GG & $110(68.3 \%)$ & $131(76.6 \%)$ & \\
\hline $\mathrm{GA}+\mathrm{AA}$ & $51(31.7 \%)$ & $40(23.4 \%)$ & \\
\hline
\end{tabular}

CAD indicates coronary artery disease, defined as $\geq 50 \%$ diameter stenosis compared with reference vessel diameter by coronary angiography. 
although there was no difference in patients in the $\geq 60$ years age group $(27.7 \%$ versus $25.2 \%, P=0.644)$.

In a similar fashion, in the subgroups of nondiabetic and nonhypertensive patients, the A allele carriers were significantly more frequent in the CAD patients than in the controls compared with the diabetes and hypertensive patient subgroups (Table IV). We also observed a slightly higher prevalence of G395A polymorphisms in the nondyslipidemia and nonsmoking subgroups, although the difference did not reach statistic significance, but not in the dyslipidemia and smoking patient subgroups (Table IV).

KLOTHO G395A allele carrier state as an independent risk factor of coronary artery disease: Multivariate analysis using a logistic regression model was performed to evaluate KLOTHO G395A allele carrier state as an independent risk factor of coronary artery disease. This model included age, sex, hypertension, diabetes, dyslipidemia, smoking, and BMI along with KLOTHO G395A carrier state, which were significant risk factors in the univariate analysis and traditional risk factors of CAD. The KLOTHO G395A carrier state was an independent risk factor of coronary artery disease (OR 1.712, 95\% CI [1.066-2.749], $P=0.026$ ) (Table V).

Association of KLOTHO G395A polymorphism with coronary artery calcification: The incidence of patients who had coronary calcification in at least 1 coronary artery was $51.2 \%$ (222/434). Patients with a calcified coronary artery were significantly more common in the CAD patients than in those without CAD; $67.4 \%$ (155/230) versus $32.8 \%(67 / 204), P<0.0001$.

The allele frequency (GG, GA, AA) was not different between the coronary calcification group and noncalcification group; $74.4 \%, 22.4 \%, 3.2 \%$ versus $73.6 \%, 23.6 \%, 2.8 \%$, respectively $(P=0.961)$. The frequency of the G395A allele carrier state (GA+AA) was similar between the two groups; $25.5 \%$ in the calcification group and $26.4 \%$ in the noncalcification group $(P=0.842)$. However, in renal insufficiency patients with an eGFR $<50 \mathrm{~mL} /$ minute $/ 1.73 \mathrm{~m}^{2}$, the frequency of G395A polymorphism was significantly higher in those with cal-

Table V. Risk Factors for Coronary Artery Disease by Multiple Logistic Regression Analysis

\begin{tabular}{lcrr}
\hline Variable & OR & $95 \%$ CI & \multicolumn{1}{c}{$P$} \\
\hline Klotho G395A, A allele carrier state & 1.712 & $1.066-2.749$ & 0.026 \\
Age & 1.055 & $1.035-1.075$ & $<0.001$ \\
Male sex & 2.059 & $1.292-3.280$ & 0.002 \\
Hypertension & 1.307 & $0.837-2.041$ & 0.239 \\
Diabetes & 1.413 & $0.893-2.236$ & 0.140 \\
Dyslipidemia & 1.599 & $1.046-2.443$ & 0.030 \\
Smoking & 1.849 & $1.092-3.132$ & 0.022 \\
BMI $\left(\mathrm{Kg} / \mathrm{m}^{2}\right)$ & 0.999 & $0.932-1.070$ & 0.969 \\
\hline
\end{tabular}

OR indicates odds ratio; CI, confidence interval; and BMI, body mass index. 


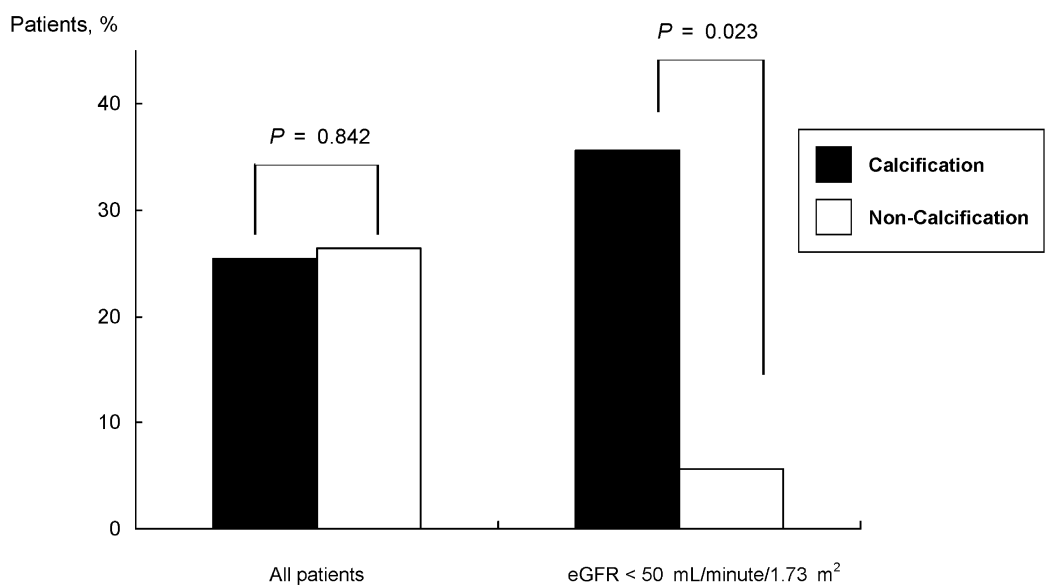

Figure. G395A mutant allele carrier (GA+AA) state according to coronary artery calcification.

cification than in the noncalcification group; 15/42(35.7\%) versus $1 / 18(5.6 \%)$, respectively ( $P=0.023$, by Fisher's exact test) (Figure). Using multiple logistic regression analysis, age (OR 1.072, 95\% CI [1.049-1.094], $P<0.001)$, diabetes (OR 1.983, 95\% CI [1.218-3.229], $P=0.006$ ), and presence of CAD (OR 5.009, 95\% CI [3.191-7.864], $P<0.001)$ were identified as the risk factors of coronary arterial calcification, while the KLOTHO A allele carrier state was not a risk factor.

\section{Discussion}

In the present study, we demonstrated that the $-395 \mathrm{~A}$ allele carrier state was significantly more common in the CAD patients than in the controls $(30.2 \%$ versus $21.5 \%, P=0.039$ ) and was an independent risk factor of coronary artery atherosclerotic stenosis by multiple logistic regression analysis. We also found, for the first time to the best of our knowledge, that coronary calcification was not related with the KLOTHO gene G395A polymorphism.

Human KLOTHO G395A polymorphism has been reported to influence promoter function, while G-->A substitution affected KLOTHO expression and influenced bone density. ${ }^{6}$ It was demonstrated that low bone mineral density may be associated with arterial calcification and generalized atherosclerosis, and may be an independent predictor of cardiovascular mortality. ${ }^{10)}$ The mechanism of the beneficial function of KLOTHO remains to be investigated. Recent experiments have found that endothelium-dependent vasodilation of the aorta and arterioles was impaired in heterozygote KLOTHO mice ${ }^{11)}$ and nitric oxide metabolites in urine are significantly lower in heterozygote mice. ${ }^{11)}$ This result may 
imply that the cardiovascular system could be protected by KLOTHO through endothelium-derived nitric oxide production ${ }^{11,12)}$ and its genetic variation might affect the risk of CAD. Moreover, recent publications demonstrated that KLOTHO was likely to have a role in calcium balancing on the cell surface and regulating glucose metabolism in organs. ${ }^{13,14)}$ These data may be translated into KLOTHO becoming a potential target for therapeutic intervention in metabolic disorders which are closely associated with CAD.

The first suggestion that G395A polymorphism may be related to human CAD came from the study conducted by Imamura, et al that included 197 CAD patients diagnosed by coronary angiography using CAD criteria similar to those of our study. ${ }^{8)}$ They reported that A allele carriers were significantly more common in CAD patients than in controls (29.9\% versus $19.0 \%$ ), and the frequencies were similar to our results.

The allele frequency of KLOTHO G395A polymorphism in the control group of our study was $12.2 \%$ for A allele, a finding which was consistent with other data reported in Korean ${ }^{9,15)}$ and Japanese (14.3\%) subjects, ${ }^{6}$ but somewhat lower than that in whites $\left.(19.6 \%) .{ }^{6}\right)$ This less frequent adverse allele for vascular atherosclerosis may in part explain the low prevalence of coronary arterial disease in ethnic Asians compared to whites. The data obtained in the present study were also consistent with the results of a Japanese study in which G395A polymorphism was higher in the CAD group and was an independent risk factor of CAD ${ }^{8)}$ However, another study in a Korean population showed similar G395A polymorphism frequencies between CAD and control patients, in contrast to our results. ${ }^{9)}$ The reason for this discrepancy, we believe, may result from their relatively smaller sample size compared to ours.

In the subgroup of relatively younger patients $\leq 60$ years old in our study, intriguingly, the A allele was more frequent in the CAD group, especially under the more strict criteria for CAD of $\geq 75 \%$ luminal stenosis. This result may imply that the KOLTHO gene variant exerts its strength in the young population rather than in older CAD patients, which supports the findings of an article reporting KLOTHO genetic polymorphism may be related with early onset occult coronary artery disease, although the allele studied in their study was KL-VS. ${ }^{\text {) }}$

In the present subgroup analysis, in the nondiabetes and nonhypertensive group, the A allele carrier was significantly frequent in CAD patients, but not in the subgroup of diabetes and hypertensive patients. There was a similar trend in the nondyslipidemic and nonsmoking subgroup, although it failed to reach statistical significance. We believe these results may be derived and caused by the more potent impact of traditional coronary risk factors on CAD than the genetic background of KLOTHO. Therefore, it may be postulated that the KLOTHO gene mutation may have had its effect on the less ill patient group. 
The strengths of our study include the accurate estimation of coronary artery stenosis and calcification by performing coronary angiography in all the study patients as compared with the indirect evaluation of CAD status with thalium 201 scintigraphy in another study, ${ }^{5)}$ and the relatively large scale compared with that of other similar studies involving Japanese and Koran patients. ${ }^{8,9)}$ We found that the coronary calcification determined by cine-angiography and fluoroscopic evaluation was not related to the KLOTHO G395A variant. A query may be raised regarding our method of evaluating the coronary calcification. However, a previous article reported the fluoroscopy guided determination of coronary calcification was a highly specific, easy to perform, and inexpensive method. ${ }^{16)}$ Moreover, we evaluated coronary calcification in combination with cine-angiography in at least 3 other images examined by 2 interventional experts at our hospital.

We have demonstrated here for the first time that a KLOTHO gene mutation is a new risk factor of CAD, and described its relationship with coronary arterial calcification in a Korean population. Our data may be further important evidence suggesting an association between genetic background and CAD, at least in this Asian ethnic group.

Conclusions: In conclusion, the KLOTHO gene G395A allele carrier state was identified as a novel risk factor of coronary artery disease, but was not associated with coronary artery calcification in this Korean population.

\section{REFERENCES}

1. Kurosu H, Yamamoto M, Clark JD, et al. Suppression of aging in mice by the hormone Klotho. Science 2005; 309: 1829-33.

2. Kuro-o M, Matsumura Y, Aizawa H, et al. Mutation of the mouse klotho gene leads to a syndrome resembling ageing. Nature 1997; 390: 45-51.

3. Arking DE, Krebsova A, Macek M Sr, et al. Association of human aging with a functional variant of klotho. Proc Natl Acad Sci USA 2002; 99: 856-61.

4. Arking DE, Atzmon G, Arking A, Barzilai N, Dietz HC. Association between a functional variant of the KLOTHO gene and high-density lipoprotein cholesterol, blood pressure, stroke, and longevity. Circ Res 2005; 96: 412-8.

5. Arking DE, Becker DM, Yanek LR, et al. KLOTHO allele status and the risk of early-onset occult coronary artery disease. Am J Hum Genet 2003; 72: 1154-61.

6. Kawano K, Ogata N, Chiano M, et al. Klotho gene polymorphisms associated with bone density of aged postmenopausal women. J Bone Miner Res 2002; 17: 1744-51.

7. Ogata N, Matsumura Y, Shiraki M, et al. Association of klotho gene polymorphism with bone density and spondylosis of the lumbar spine in postmenopausal women. Bone 2002; 31: 37-42.

8. Imamura A, Okumura K, Ogawa Y, et al. Klotho gene polymorphism may be a genetic risk factor for atherosclerotic coronary artery disease but not for vasospastic angina in Japanese. Clin Chim Acta 2006; 371: 66-70.

9. Rhee EJ, Oh KW, Lee WY, et al. The differential effects of age on the association of KLOTHO gene polymorphisms with coronary artery disease. Metabolism 2006; 55: 1344-51.

10. Hamerman D. Osteoporosis and atherosclerosis: biological linkages and the emergence of dual-pur- 
JO, ET AL Int Heart J

pose therapies. QJM 2005; 98: 467-84. (Review)

11. Nagai R, Saito Y, Ohyama Y, et al. Endothelial dysfunction in the klotho mouse and downregulation of klotho gene expression in various animal models of vascular and metabolic diseases. Cell Mol Life Sci 2000; 57: 738-46.

12. Saito Y, Yamagishi T, Nakamura T, et al. Klotho protein protects against endothelial dysfunction. Biochem Biophys Res Commun 1998; 248: 324-9.

13. Cha SK, Ortega B, Kurosu H, Rosenblatt KP, Kuro-o M, Huang CL. Removal of sialic acid involving Klotho causes cell-surface retention of TRPV5 channel via binding to galectin-1. Proc Natl Acad Sci USA 2008; 105: 9805-10.

14. Kurosu H, Choi M, Ogawa Y, et al. Tissue-specific expression of betaKlotho and fibroblast growth factor (FGF) receptor isoforms determines metabolic activity of FGF19 and FGF21. J Biol Chem 2007; 282: 26687-95

15. Kim Y, Kim JH, Nam YJ, et al. Klotho is a genetic risk factor for ischemic stroke caused by cardioembolism in Korean females. Neurosci Lett 2006; 407: 189-94.

16. Bartel AG, Chen JT, Peter RH, Behar VS, Kong Y, Lester RG. The significance of coronary calcification detected by fluoroscopy. A report of 360 patients. Circulation 1974; 49: 1247-53. 